\title{
Electrical Activation of Polymer Reny1002H Surface with Laser Irradiation for Molded Interconnect Devices
}

\author{
Gang Huang ${ }^{1}$, Hailun Wang1, Zhongke Wang*2, Paul Lim², Yin Chi Wan², Yujie Zhou² \\ ${ }^{1}$ Electrical and Information Engineering College, Quzhou University, No.78 N. Jiuhua Rd, Quzhou, \\ 324000 China \\ 2 Singapore Institute of Manufacturing Technology, 2 Fusionopolis Way 138634 Singapore \\ *Corresponding author's e-mail: zkwang@simtech.a-star.edu.sg
}

\begin{abstract}
Polymers Reny 1002H blended with 3\% by weight Merck Iriotec 8841 (dicopper hydroxide phosphate) after injection molding was irradiated using a $\mathrm{CO}_{2}$ laser, and followed by copper electroless plating. It has been clearly observed that the surface activation was determined by the interaction level between laser and resin material, namely laser power, scanning speed and line hatching pitch. With increasing laser scanning speed, naturally the modification level was reduced, and electrical resistance was reduced. Line pitch played a similar function to scanning speed. At increasing line hatch spacing, close or larger than the laser spot size, the surface was not able to be modified uniformly. Electrical resistance decreased at a higher modification level with high laser power. SEM analysis disclosed that the copper plated surface was a porous surface structure. The lowest electrical resistance 0.043 Ohms at laser power of $18 \mathrm{~W}$, spot size of $200 \mu \mathrm{m}$, hatching pitch of $150 \mu \mathrm{m}$ and scanning speed of $150 \mathrm{~mm} / \mathrm{s}$. The calculated electrical resistivity was $1.62 \times 10^{-7} \Omega \mathrm{m}$ which was one order of magnitude lower than the bulk copper of $1.72 \times 10^{-8} \Omega \mathrm{m}$ due to the porous structure disclosed under scan electron microscope (SEM) and the electron dispersive X-ray spectroscopy (EDX) measurements.
\end{abstract}

DOI: 10.2961/jlmn.2021.01.2012

Keywords: surface electrical activation, polymer Reny1002H, selective laser activation, electroless plating, 3D Molded Interconnected Device, direct laser structuring

\section{Introduction}

Three-Dimensional Molded Interconnect Devices (3D MID) are devices integrating mechanical, electric and electronical functions, which having been realized 30 years ago [1]. 3D MID makes it possible to add electronic functionality on three-dimensional objects without the use of conventional cable wiring. Circuit layouts have been created on complex three-dimensional carrier structures using Leiterplatten-Kopierfräsen (LPKF) laser direct structuring (LDS) since 1997 [2]. Structuring finest lines down to approximately $20 \mu \mathrm{m}$ has been achieved through laser supported additive metallization of different thermoplastic materials for 3D MID nearly 20 years ago [3]. 3D MID from prototyping to mass production with LDS has been obtained 15 years ago [4]. The employment of 3D MID has dramatically increased in the past decade as they offer flexibility on the design and function of free form systems or elements. Up to now, 3D MID have been employed in numerous applications besides automotive [5], such as telecommunications and antennas [6,7], self-biased circulator [8], stretchable electronics [9], sensor technology [10,11], medical technology [12] etc.

To date, LDS has been realized to be an essential process for MID production [13,14]. LDS provides a method of reducing part count, light weighting and increasing component density in electronic devices $[2,15,16]$. This is achieved by allowing for the plating of current carrying pathways onto the surface of injection molded structural components. First, a plastic is blended with an electroless plating seed compound is injection molded into a structural component. The conductive circuit paths are selectively laser structured on the part surface $[17,18]$. This part is then plated using an electroless plating process, with plating only occurring on surfaces that are laser structured. This allows for the elective plating of only the desired conductive paths $[19,20]$. The process is additionally advantageous for the electronics industry because it is also relatively easy to automate.

Although LDS is state of the art and the most commercialized process for MID production, the technology has found niche but significant applications in the manufacture of mobile devices and in the automotive industry. However, LDS is a technique relying on using precursors mixed in a polymer $[2,3,21]$. These precursor additives are activated during the laser writing process converting them into a catalyst for electroless deposition of the metal matrix. So, the polymer substrate plays a crucial role in 3D MID technology. Polymer based composite are the primary choice to be used as substrate, which has been comprehensively reviewed recently regarding the requirement criteria for manufacturing a polymer substrate and the main surface modification techniques used to enhance the polymer substrate for 3D MID manufacturing [22]. The reviewed polymers have covered from thermoplastics, modified thermoplastics to thermoplastics composites. The polymer materials should enable good flexibility in production from macro to micro-MID products, high fracture toughness when subjected to mechanical loading, and lightweight. 
To explore the possible new polymers employed and copper precursors for 3D MID products, in this study, Mitsubishi polymers Reny $1002 \mathrm{H}$ blended with 3\% by weight Merck Iriotec 8841 (dicopper hydroxide phosphate) after injection molding was irradiated using a $\mathrm{CO}_{2}$ laser and copper electroless plated for potential 3D MID manufacturing.

\section{Experiments}

\subsection{Polymer selection}

A survey of the applications of 3D MID components showed that Nylon blends were highly desirable given that the objective of 3D MID manufacturing was to transfer circuit carrier paths to a structural polymer component. As such the plastic of choice should be strong, has good chemical resistance and good thermal resistance and conductivity. Nylon PA6-GF30 blended with electroless plating seed compound has been selected, Mitsubishi polymers Reny $1002 \mathrm{H}$ blended with 3\% by weight Merck Iriotec 8841, a copper phosphate/tin oxide mixture.

The injection molding was carried out using an industrial reciprocating screw injection molding machine. The polymers were molded into plates that were then cut to size for laser surface activation.

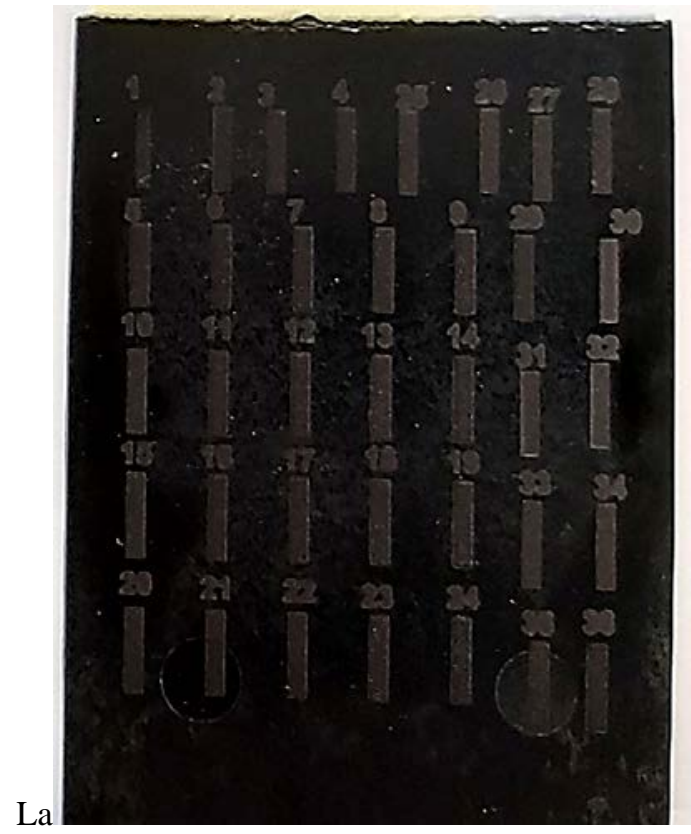

Fig. 1 Sample after laser irradiation under various laser parameter settings.

\section{2 ser surface activation}

Laser surface activation through laser direct structuring has the primary effect of creating a porous, convoluted surface that exposes large quantities of the embedded seed material particles. These particles serve as nucleation sites for the electroless plating process. The creation of this convoluted surface and the attendant exposure of a local abundance of seed materials particles allows for electroless plating to 'grow' layer of conductive metal.

A $\mathrm{CO}_{2}$ laser used in laser irradiation was Synrad Firestar Ti60. The laser has a full power of $60 \mathrm{~W}$, which was modulated at different pulse repetition rate from $1 \mathrm{KHz}$ to
$100 \mathrm{KHz}$. The beam spot was $0.2 \mathrm{~mm}$ at focus under a galvo scanner focal lens. Laser beam was scanned at different hatching pitch from $0.1 \mathrm{~mm}$ to $0.25 \mathrm{~mm}$. A rectangular area of $10 \mathrm{~mm}$ by $1 \mathrm{~mm}$ area was scanned for each parameter settings, as shown in Figure 1. The choice of $\mathrm{CO}_{2}$ laser is firstly because it is strongly absorbed by nearly all organic polymers. Secondly, $\mathrm{CO}_{2}$ lasers are versatile, inexpensive and widely used in industry.

\subsection{Electroless copper plating}

Laser surface irradiated sample was first cleaned with an alkaline cleaner. The sample was then rinsed with distilled water. The cleaning process was then repeated with sulphuric acid. The sample was then treated with a palladium activator. The sample was finally plated with a copper sulfate solution. The detailed procedures are shown below.

1) Place sample in Alkali Etching Solution at $75^{\circ} \mathrm{C}$ for 30 min for decreasing.

2) DI water rinse

3) Dip sample in $10 \%$ Sulphuric Acid Solution to remove surface oxide.

4) DI water rinse

5) Place samples in Activator solution for 3 min to activate the surface.

6) DI water rinse

7) Place samples in Electroless Copper solution at $50^{\circ} \mathrm{C}$ for 1 hour.

8) DI water rinse

9) Place sample in Copper Anti-tarnish Solution for 10 $\min$

10) DI water rinse

11) Blowing gas drying with hairdryer

Copper was plated through the following reaction:

$$
2 \mathrm{HCHO}+4 \mathrm{OH}^{-} \rightarrow 2 \mathrm{HCOO}^{-}+2 \mathrm{H}_{2} \mathrm{O}+{ }_{\mathrm{H} 2}(\uparrow)+2 \mathrm{e}^{-}
$$$$
\mathrm{Cu}^{2+}+2 \mathrm{e}^{-} \rightarrow \mathrm{Cu} \text { (metal) }
$$

A series of laser irradiation areas were produced with the optimal performing parameter setting. The samples were plated in batch. Electroless copper plating setup is shown in Figure 1. Some of the samples in this batch were sectioned to determine plating thickness and to facilitate a calculation of resistivity of the plated layer.

\section{Results and discussion}

\subsection{Laser activation analysis}

After laser irradiation, a porous structure was produced on blended Reny surface, as shown in Figure 2. Reny ${ }^{\circledR}$ $1002 \mathrm{H}$ is a polyamide MXD6 grade reinforced with 30\% glass fibers. Under IR $\mathrm{CO}_{2}$ laser beam irradiation, the polymer absorbed the incident photon energy and converted it into heat through photo-thermal and photo-degradation mechanisms [23-25]. The transition of molecules of polyamide from the ground state to the excited state after absorb photon energy leading to chain breaks and the formation of free radical in photo-degradation mechanism. For the long-wavelength laser, the IR laser couples to $\mathrm{C}-\mathrm{C}$ bonds present in the carbon precursor and provides efficient photothermal heating [25]. The carbon in the polyamide oxidized to form $\mathrm{CO}_{2}$ which emerged as bubbles, leading to a porous foam structure. The glass fibers melted and resolidified to form worm-like structures uniformly distrib- 
uted in the matrix under laser irradiation, as shown from SEM image in Figure 2.

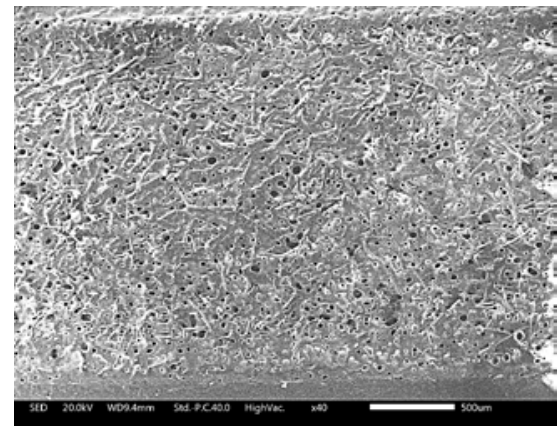

Fig. 2 Porous surface morphology generated after laser irradiation under power of $60 \mathrm{~W}$, speed of $1 \mathrm{~m} / \mathrm{s}$ and line pitch spacing of $0.1 \mathrm{~mm}$

Surface activation was dependent on the interaction between laser and resin materials, which were further determined by the laser power, scanning speed and line hatching pitch. The pigment Iriotec ${ }^{\circledR}$ 8841, dicopper hydroxide phosphate $\left(\mathrm{Cu}_{2} \mathrm{HO}_{5} \mathrm{P}\right)$ in the Reny matix decomposed under the sufficient laser irradiation. Energy Dispersive XRay (EDX) measurements have shown that at lower laser power or high scanning speed, the surface could not be able to activate due to the insufficient interaction between laser and Reny. Figure 3 shows that no copper seeds were synthesized in laser irradiation at a high speed of $1 \mathrm{~m} / \mathrm{s}$ and lower of $24 \mathrm{~W}$ with line pitch of $0.1 \mathrm{~mm}$. High power and/or slow speed were necessary to synthesize copper seed by laser irradiation under high interaction levels. Dicopper hydroxide phosphate decomposed, resulting in copper atom formation while Reny matrix melted. For example, laser irradiation under speed of $0.15 \mathrm{~mm} / \mathrm{s}$ and power $18 \mathrm{~W}$, as shown in Figure 4. It indicates that copper atoms and/or ions were produced as pre precursors for electroless plating.

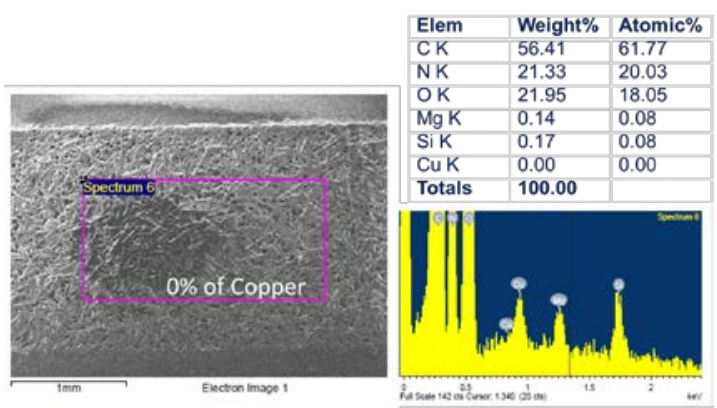

Fig. 3 EDX measurements showing no copper seeds synthesized in laser irradiation at a high speed of $1 \mathrm{~m} / \mathrm{s}$ and lower of $24 \mathrm{~W}$ with line pitch of $0.1 \mathrm{~mm}$.
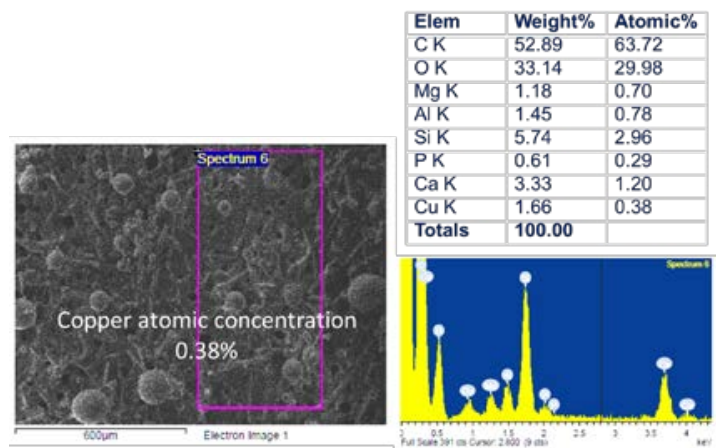

Fig. 4 EDX measurements showing the copper seeds synthesized by laser irradiation at a slower speed of $0.15 \mathrm{~m} / \mathrm{s}$ and lower of $18 \mathrm{~W}$ with line pitch of $0.15 \mathrm{~mm}$.

\subsection{Electrical resistance analysis}

The optical image of the results of electroless plating after laser irradiation were shown in Figure 5. It indicates that the plating was varied with the laser irradiation conditions. The electrical resistance of the copper plated samples was measured with a digit multimeter (Agilent 34401A). The resistance was mostly more or less 0.05 ohms under the laser irradiation conditions investigated. The electrical resistance was plotted in Figures 6, 7 and 8. Electrical resistance decreased at increasing laser power as stronger modification of Reny surface was made at a given speed with a high level of laser power. Open circuits were obtained when power was below $30 \mathrm{~W}$ as shown in Figure 6 .

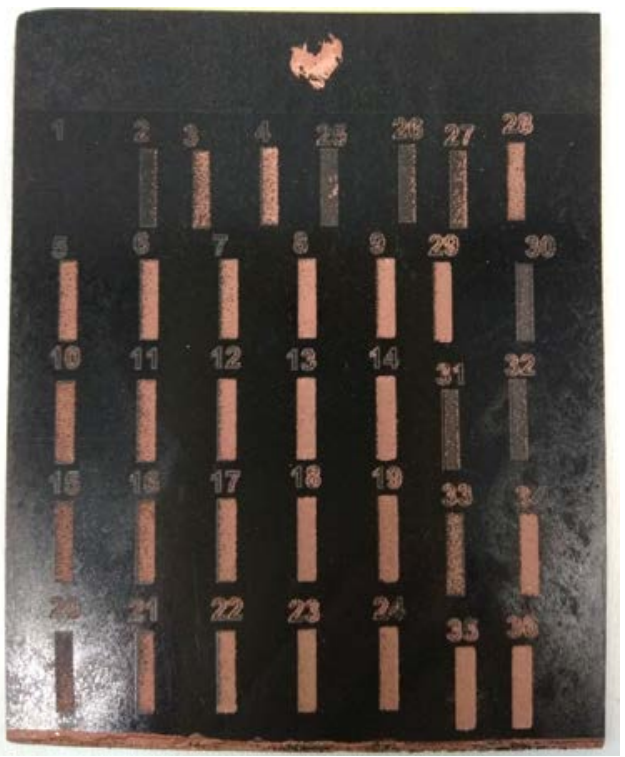

Fig. 5 Electroless copper plating results from Reny irradiated under various laser conditions.

However, the power level is not the only role to determine the modification level for creation of electrical conductivity. Scanning speed is another important role to determine the electrical resistance. Figure 7 shows the electrical resistance obtained a lower power level of $24 \mathrm{~W}$. It indicates that slow speed may make a compensation to the low power as a longer interaction time at slow speed may achieve a similar modification level as high power. With 
increasing laser scanning speed, naturally the modification level was reduced due to the reduced interaction time or pulse number at high scanning speed, and thus the electrical resistance was reduced as shown in Figure 7.

Line pitch will determine that which area could be laser modified. At increasing line hatch spacing, especially close or larger than the laser spot size, the area between two lines may not be well modified under laser irradiation. The surface was not able to be modified uniformly at larger line space. Figure 8 shows that the electrical resistance was increased suddenly and tended to open circuits when line pitch larger than the spot size. Line pitch similarly is another factor ton influence modification level and uniformity under laser irradiation.

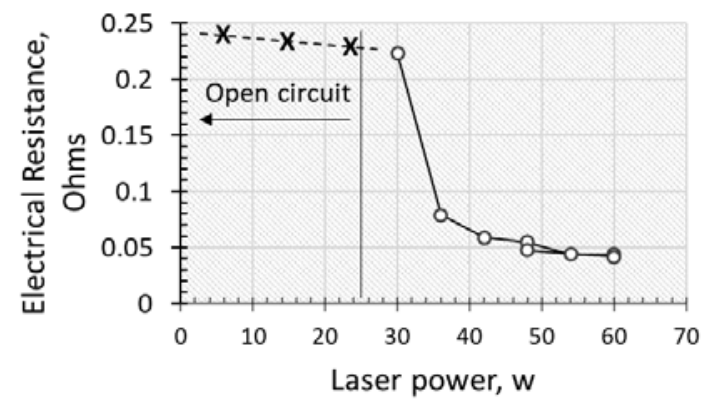

Fig. 6 Electrical resistance decreasing with laser power. Scanning speed at $1 \mathrm{~m} / \mathrm{s}$, line hatch spacing at $0.1 \mathrm{~mm}$.

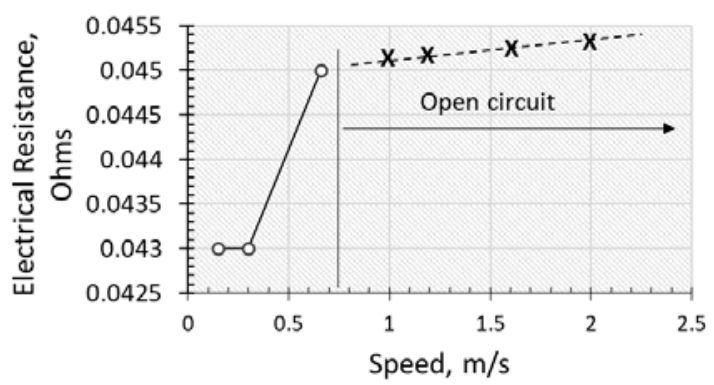

Fig. 7 Electrical resistance increasing with scanning. Laser power of $24 \mathrm{~W}$, line hatch spacing at $0.15 \mathrm{~mm}$.

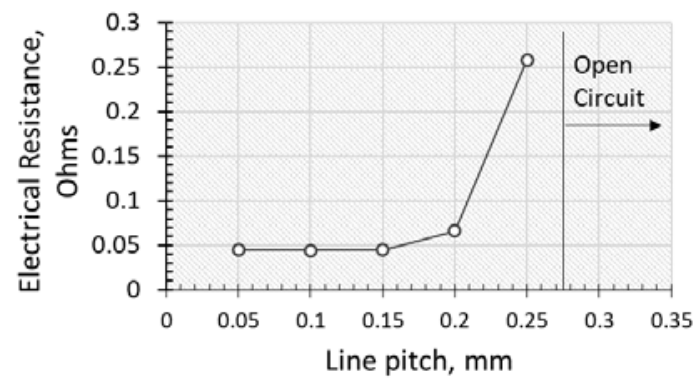

Fig. 8 Electrical resistance increasing at larger line hatch spacing. Laser power of $60 \mathrm{~W}$, scanning speed of $1 \mathrm{~m} / \mathrm{s}$.

The study revealed that under the investigated laser irradiation conditions as shown in Figure 6-8, the lowest resistance was about 0.043 ohms. Electroless plating generated a relatively uniform copper layer on the laser modified area as shown in Figure 9. As a comparison, Figure 10 shows a not well copper plated sample due to the insuffi- cient plating seeds synthesized in laser surface irradiation modification. The plated copper appeared as islands which are isolated and couldn't form a close loop for electrical circuit.

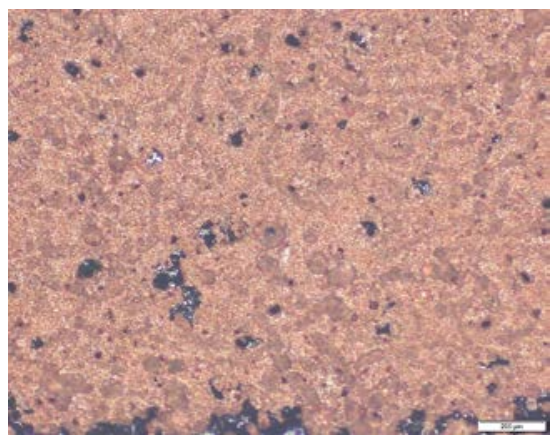

Fig. 9 Surface morphology under optical microscope after copper plating for sample obtained under laser power $18 \mathrm{~W}$, scanning speed of $0.15 \mathrm{~m} / \mathrm{s}$ and line hatch spacing of $0.15 \mathrm{~mm}$.

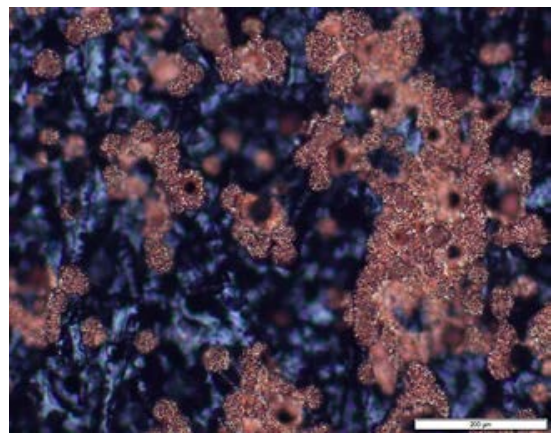

Fig. 10 Surface morphology under optical microscope after copper plating for sample obtained under laser power of $24 \mathrm{~W}$, scanning speed of $1 \mathrm{~m} / \mathrm{s}$ and line hatching pitch of $0.1 \mathrm{~mm}$.

\subsection{Electrical resistivity analysis}

Above experimental study all is on the electrical resistance after electroless copper plating of laser irradiated surfaces. After electroless plating, the electrical resistivity could be measured by knowing the copper layer length, cross sectional area and resistance of the sample. Based on the results achieved above, parameter settings as laser power of $18 \mathrm{~W}$, scanning speed of $0.15 \mathrm{~m} / \mathrm{s}$ and line hatch spacing of $0.15 \mathrm{~mm}$ was used to characterize the electrical resistivity of the copper plated polymer after laser irradiation. Figure 12 shows a cross section of the plated surface layer. Figure 13 shows the top surface morphology of copper plated area observed under SEM system. A porous structure with microholes was formed after electroless copper plating. The measured thickness of the plated copper was $0.0887 \mathrm{~mm}$ from the average of five measurements. The electrical resistivity is given by $\rho=\mathrm{R} A / \mathrm{L}$.

Where $\rho, R, A$ and $L$ stand for the electrical resistivity, the measured electrical resistance, the cross-section area size, and the length of the plated copper layer, respectively.

The electrical resistivity calculated was to be $1.62 \times 10^{-7}$ $\Omega \mathrm{m}$, which is about an order of magnitude higher than a theoretical copper plate of the same dimension of copper of $1.72 \times 10^{-8} \Omega \mathrm{m}$. The most obvious reason for this higher resistivity could be due to the pitting and the porosity of the plated surface as seen in optical image Figure 11 and SEM 
image Figure 12. The laser activated surface is not fully covered by copper due to the porous structure. Further analysis has been performed using EDX measurements. Figure 13 disclosed from EDX analysis that copper concentration was about $90 \%$ in weight. There were about $10 \%$ in weight impurity in the copper plated layer. The holes’ composition is remained polymer nature without copper detected, as EDX measurements indicated in Figure 14.

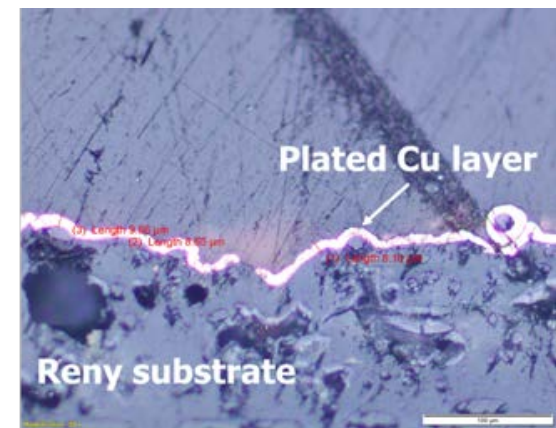

Fig. 11 A cross-section of the plated surface observed under optical microscope.

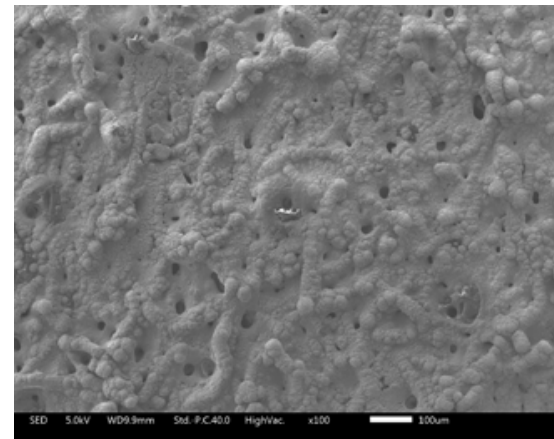

Fig. 12 SEM image showing a porous microstructure of the electroless plated surface.

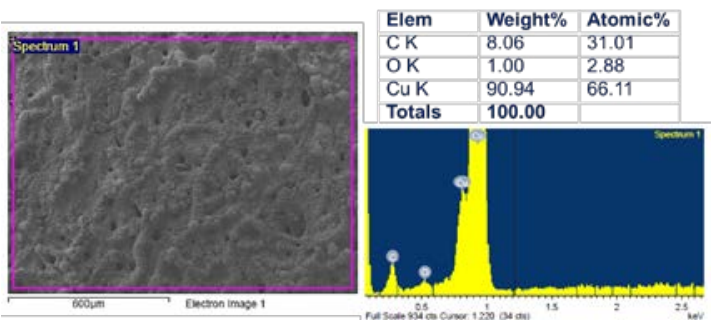

Fig. 13 The porous copper layer with a weight about 90.94\% after electroless plating for laser setting: power of $18 \mathrm{~W}$, scanning speed of $0.15 \mathrm{~m} / \mathrm{s}$ and line hatch spacing of $0.15 \mathrm{~mm}$.

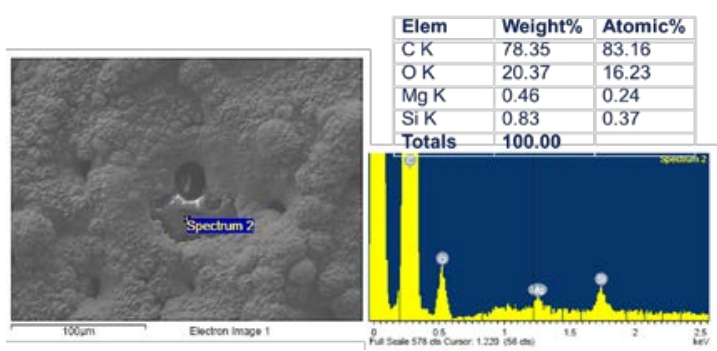

Fig. 14 EDX measurements showing free of copper in the microholes in electroless copper plated layer. Laser setting: power of 18 $\mathrm{W}$, scanning speed of $0.15 \mathrm{~m} / \mathrm{s}$ and line hatch spacing of $0.15 \mathrm{~mm}$.

\section{Conclusion}

Direct $\mathrm{CO}_{2}$ laser surface activation of polymer Reny 1002H blended with 3\% by weight Merck Iriotec 8841 (a copper phosphate/tin oxide mixture) substrates employed in the manufacture of 3D MID devices has been demonstrated successfully. Copper seeds synthesis under laser irradiation for electroless copper plating was a multiple factor interactive interaction result, laser power, scanning speed and line pitch. The lowest electrical resistance $0.043 \mathrm{Ohms}$ at laser power of $18 \mathrm{~W}$, spot size of $200 \mu \mathrm{m}$, hatching pitch of 150 $\mu \mathrm{m}$ and scanning speed of $150 \mathrm{~mm} / \mathrm{s}$. The calculated electrical resistivity was $1.62 \times 10^{-7} \Omega \mathrm{m}$ which was one order of magnitude lower than the bulk copper of $1.72 \times 10^{-8} \Omega \mathrm{m}$ due to the porous structure disclosed under SEM and EDX measurements.

\section{Acknowledgments}

The research work was supported by A-Star Research Agency, Singapore Institute of Manufacturing Technology (SIMTech) under project No. C16-M-036.

\section{References}

[1] K. Feldmann and J. Franke: Proc. 11th IEEE Int. Electron. Manuf. Technol. Symp., (1991) 320.

[2] J. Franke: "Three-Dimensional Molded Interconnect Devices (3D-MID): Materials, Manufacturing, Asembly, and Applications for Injection Molded Circuit Carriers” (Hanser, Munich, 2014) p.65.

[3] M. Hüske, J. Kickelhain, J. Müller, and G. Eßer: Proc 3rd LANE, (2001) 95.

[4] N. Heininger, W. John, and H.-J. Boßler: Proc 6th MID, (2004).

[5] D. Moser and J. Krause: "3-D-MID - Multifunctional Packages for Sensors in Automotive Applications”, in Advanced Microsystems for Automotive Applications edited by J. Valldorf and W. Gessner, (SpringerVerlag, Berlin/Heidelberg, 2006) p.369.

[6] K. Becker and T. Braun: A. Neumann, A. Ostmann, M. Koch, V. Bader, R. Aschenbrenner, H. Reichl, IEEE Trans. Electron. Packag. Manuf., 28, (2005) 291.

[7] J. Hoerber, J. Glasschroeder, M. Pfeffer, J. Schilp, M. Zaeh, and J. Franke: Procedia CIRP, 17, (2014) 806.

[8] V. Laur, J.-L. Mattei, G. Vérissimo, P. Queffelec, R. Lebourgeois, and J.-P.e Ganne: J. Magn. Magn. Mater., 404, (2016) 126.

[9] C. Votzke, S. Member, U. Daalkhaijav, Y. Mengüç, and M. L. Johnston: IEEE Sens. J., 19, (2019) 3832

[10] A. Nadeem, M. A. Hussain, O. Owais, A. Salam, S. Iqbal, and K. Ahsan: Comput. Netw, 83, (2015) 363.

[11] M. P. Schmidt, A. Oseev, C. Engel, A. Brose, B. Schmidt, and S. Hirsch: J. Sens. Sens. Syst., 5, (2016) 55.

[12] H. Peng, K. Wang, and Z. Huang: Mater. Manuf. Process., 34, (2019) 256.

[13] A. Islam, H. N. Hansen, P. T. Tang, and J. Sun: Int. J. Adv. Manuf. Technol., 42, (2009) 831.

[14]B. Bachy, R. Süß-Wolf, L. Wang, Z. Fu, N. Travitzky, P. Greil, and J. Franke: Adv. Eng. Mater., 20, (2018) 1700824 .

[15] P. Amend, C. Pscherer, T. Rechtenwald, T. Frick, and M. Schmidt: Phys. Procedia., 5, (2010) 561. 
[16] S. Balzereit, F. Proes, V. Altstädt, and C. Emmelmann: Addit. Manuf., 23, (2018) 347.

[17] K. Kuhmann, R. Dorfler, and G. W. Ehrenstein: Kunst. Plast. Eur., 91, (2001) 15.

[18] K. Feldmann and A. Kunze: Kunst. Plast. Eur., 94, (2004) 17.

[19] T. Leneke, S. Hirsch, and B. Schmidt: Circuit World., 35, (2009) 23.

[20] Y. Lu, H. Y. Yun, M. Vatani, H. C. Kim, and J. W. Choi: J. Mech. Sci. Technol., 29, (2015) 5377.

[21] K. Ratautas, A. Jagminienè, I. Stankevičienė, M. Sadauskas, E. Norkus, and G. Račiukaitis: Results Phys, 16, (2020) 102943.

[22] J. Tengsuthiwat, M. R. Sanjay, S. Siengchin, and C. Pruncu: Polymers, 12, (2020) 1408.

[23] M. Inagaki, S. Harada, T. Sato, T. Nakajima, Y. Horino, and K. Morita: Carbon, 21, (1989) 253.

[24] J. Lin, Z. Peng, Y. Liu, F. Ruiz-Zepeda, R. Ye, E. L.G. Samuel, M. J. Yacaman, B. I. Yakobson, and J. M. Tour: Nat. Commun., 5, (2014) 5714.

[25] N. Ahmed, S. Darwish, and A. M. Alahmari: Mater. Manuf. Process., 31, (2016) 1121.

(Received: June 22, 2020, Accepted: March 14, 2021) 